Supporting Information for Publication

\title{
Simultaneous Extraction of Sulfur and Nitrogen Compounds from Model Diesel Fuel using Neoteric Green Solvents
}

\author{
Hurun E. Suhaimi ${ }^{1,2}$, Hanee F. Hizaddin ${ }^{* 1,2}$, Irfan Wazeer $^{3}$, Lahssen El-Blidi ${ }^{3}$, Mohd A. Hashim ${ }^{1,2}$, \\ Mohamed K. Hadj-Kali*3 \\ ${ }^{1}$ Department of Chemical Engineering, Universiti Malaya, Kuala Lumpur, Malaysia \\ ${ }^{2}$ University of Malaya Centre for Ionic Liquids (UMCiL), Universiti Malaya, Kuala Lumpur, Malaysia \\ ${ }^{3}$ Chemical Engineering Department, King Saud University, P.O Box 800, Riyadh 11421, Saudi Arabia \\ *Corresponding authors: hanee@um.edu.my ; mhadjkali@,ksu.edu.sa
}




\section{Nomenclature}

\begin{tabular}{|c|c|}
\hline AceA & acetic Acid \\
\hline BT & benzothiophene \\
\hline$[\mathrm{BDMIM}]\left[\mathrm{BF}_{4}\right]$ & 1-Butyl-2,3-dimethylimidazolium tetrafluoroborate \\
\hline$[\mathrm{BMIM}]\left[\mathrm{BF}_{4}\right]$ & 1-butyl-3-methylimidazolium tetrafluoroborate \\
\hline$[\mathrm{BMIM}][\mathrm{Br}]$ & 1-butyl-3-methylimidazolium bromide \\
\hline$[\mathrm{BMIM}][\mathrm{Cl}]$ & 1-butyl-3-methylimidazolium chloride \\
\hline$[\mathrm{BMIM}]\left[\mathrm{PF}_{6}\right]$ & 1-butyl-3-methylimidazolium hexafluorophosphate \\
\hline $\mathrm{CA}$ & citric Acid \\
\hline $\mathrm{ChCl}$ & choline chloride \\
\hline$\left[\left(\mathrm{CH}_{2}\right)_{4} \mathrm{SO}_{3}[\mathrm{HMIM}][\mathrm{Tos}]\right.$ & 1-methyl-3-(4-sulfonic acid butyl) imidazole p-toluenesulfonic acid \\
\hline$\left[\left(\mathrm{CH}_{2}\right)_{4} \mathrm{SO}_{3}[\mathrm{HMIM}]\left[\mathrm{ZnCl}_{3}\right]\right.$ & 1-methyl-3-(4-sulfonic acid butyl) imidazole zinc chloride \\
\hline CFPIL & carboxyl functionalized poly(ionic liquid) \\
\hline $\mathrm{CoCl}_{2}$ & Cobalt chloride \\
\hline$\left[\mathrm{C}_{2} \mathrm{mim}\right]\left[\mathrm{Et}_{2} \mathrm{PO}_{4}\right]$ & 1-ethyl-3-methylimidazolium diethylphosphate \\
\hline$\left[\mathrm{C}_{2} \mathrm{mim}\right]\left[\mathrm{NTf}_{2}\right]$ & 1-ethyl-3-methylimidazolium bis(trifluoromethylsulfonyl)imide \\
\hline$\left[\mathrm{C}_{2} \mathrm{mim}\right][\mathrm{OAc}]$ & 1-ethyl-3-methylimidazolium acetate \\
\hline$\left[\mathrm{C}_{6} \mathrm{mmpy}\right]\left[\mathrm{NTf}_{2}\right]$ & 1-hexyl-2,4dimethylpyridinium bis(trifluoromethylsulfonyl)imide \\
\hline DBT & dibenzothiophene \\
\hline EG & ethylene glycol \\
\hline [EMIM][MeSO3] & 1-ethyl-3-methylimidazolium methanesulfonate \\
\hline$[\mathrm{EMIM}]\left[\mathrm{EtSO}_{4}\right]$ & 1-ethyl-3-methylimidazolium ethyl sulfate \\
\hline$[\mathrm{EMPY}]\left[\mathrm{EtSO}_{4}\right]$ & 1-ethyl-3-methylpyridinium ethyl sulfate \\
\hline$[\mathrm{EPy}][\mathrm{BF} 4]$ & N-Ethylpyridinium Tetrafluoroborate \\
\hline GA & Glycolic Acid \\
\hline $\mathrm{HGO}$ & Heavy Gas Oil \\
\hline$[\mathrm{Hnmp}]\left[\mathrm{HSO}_{4}\right]$ & N-methylpyrrolidonium sulfuric acid \\
\hline$[\mathrm{Hnmp}]\left[\mathrm{ZnCl}_{3}\right]$ & N-methylpyrrolidonium zinc chloride \\
\hline MA & Malonic Acid \\
\hline MTPPBr & Methyltriphenylphosphonium bromide \\
\hline PAA & Phenylacetic Acid \\
\hline PEG & polyethylene glycol \\
\hline Ppm & parts per million \\
\hline PPA & Phenylpropionic Acid \\
\hline $\operatorname{PrA}$ & Propionic Acid \\
\hline TBABr & tetrabutylammonium bromide \\
\hline TBACl & tetrabutylammonium chloride \\
\hline [TBHPP $][\mathrm{Cl}]$ & tributyl(2,3-dihydroxypropyl)phosphonium chloride \\
\hline$[\mathrm{TBCEP}][\mathrm{Br}]$ & tributyl(1,2- dicarboxyethyl)phosphonium bromide \\
\hline $\mathrm{TBPBr}$ & tetrabutylphosphonium bromide \\
\hline TEACl & tetraethylammonium chloride \\
\hline THAmBr & Tetrahexylammonium bromide \\
\hline $\mathrm{TPABr}$ & Tetrapropylammonium bromide \\
\hline 3-MBT & 3-methylbenzothiophene \\
\hline 3-MT & 3-methylthiophene \\
\hline 4-MDBT & 4-methyl dibenzothiophene \\
\hline 4,6-DMDBT & 4,6-dimethyl dibenzothiophene \\
\hline
\end{tabular}




\section{List of tables}

Table S1. List of Publication on the Extraction of Sulfur or Nitrogen Compound from fuel oil

Table S2. List of Screened DESs for COSMO-RS Study

Table S3. Chemical structure of cations used in this work

Table S4. Standard deviation (STDEV) on measured solubilities of hexadecane (1) / multisolute (2) mixture with TBPBr:EG (1:2) (3) for mole fractions x: Top layer

Table S5. Standard deviation (STDEV) on measured solubilities of hexadecane (1) / multisolute (2) mixture with TBPBr:EG (1:2) (3) for mole fractions x: Bottom layer

Table S6. Standard deviation (STDEV) on measured solubilities of hexadecane (1) / multisolute (2) mixture with TBABr:EG (1:2) (3) for mole fractions x: Top layer

Table S7. Standard deviation (STDEV) on measured solubilities of hexadecane (1) / multisolute (2) mixture with TBABr:EG (1:2) (3) for mole fractions x: Bottom layer

Table S8. Distribution Coefficient (D) and extraction efficiency $(\alpha)$ of individual solutes

Table S9. Selectivity $(\mathrm{S})$ and extraction affinity $(\beta)$ of individual solutes 
Table S1. List of Publication on the Extraction of Sulfur or Nitrogen Compound from fuel oil.

\begin{tabular}{|c|c|c|c|c|}
\hline No & Organic Solvent/ IL / DES / Catalysts & Hydrocarbon & $\begin{array}{l}\text { Sulfur \& Nitrogen } \\
\text { Compound }\end{array}$ & Ref \\
\hline 1. & $\begin{array}{l}\text { Cobalt nanoparticles were introduced as a } \\
\text { novel catalyst }\end{array}$ & $\mathrm{HGO}$ & 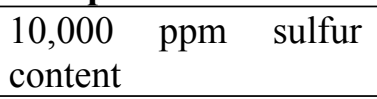 & 1 \\
\hline 2. & Catalyst: CFPIL $+\mathrm{H}_{2} \mathrm{O}_{2}$ & Iso-octane & thiophene & 2 \\
\hline 3. & $\begin{array}{l}\mathrm{AlCl}_{3}: \text { chlorinated paraffins: benzene } \\
\mathrm{AlCl}_{3} \text { : chlorinated paraffins: toluene } \\
\mathrm{AlCl}_{3}: \text { chlorinated paraffins: p-xylene } \\
\mathrm{AlCl}_{3} \text { : chlorinated paraffins: o-xylene } \\
\mathrm{AlCl}_{3}: \text { chlorinated paraffins: ethylbenzene } \\
\mathrm{AlCl}_{3} \text { : chlorinated paraffins: chlorobenzene }\end{array}$ & $\begin{array}{l}n \text {-heptane, } \\
\text { real fuel }\end{array}$ & 3-MT, BT, DBT & 3 \\
\hline 4. & $\begin{array}{l}\text { molybdenum supported on Ti-pillared } \\
\text { interlayer clay catalyst }+ \text { cyxlohexanone } \\
\text { peroxide }\end{array}$ & n-octane & DBT & 4 \\
\hline 5. & Acid-modified activated charcoal (AC) & $\begin{array}{l}\text { Cyclohexane, real } \\
\text { diesel, kerosens }\end{array}$ & DBT & 5 \\
\hline 6. & $\begin{array}{l}{[\mathrm{Hnmp}]\left[\mathrm{HSO}_{4}\right]+\mathrm{H}_{2} \mathrm{O}_{2}} \\
{\left[\left(\mathrm{CH}_{2}\right)_{4} \mathrm{SO}_{3}[\mathrm{HMIM}]\left[\mathrm{ZnCl}_{3}\right]+\mathrm{H}_{2} \mathrm{O}_{2}\right.} \\
{\left[\left(\mathrm{CH}_{2}\right)_{4} \mathrm{SO}_{3}[\mathrm{HMIM}][\mathrm{Tos}]+\mathrm{H}_{2} \mathrm{O}_{2}\right.} \\
{[\mathrm{Hnmp}]\left[\mathrm{ZnCl}_{3}\right]+\mathrm{H}_{2} \mathrm{O}_{2}}\end{array}$ & FCC Diesel Fuel & $\begin{array}{l}\text { 1,4-butane sultone } \\
\text { N-methylimidazole } \\
\text { 1-methyl-2- } \\
\text { pyrrolidone }\end{array}$ & 6 \\
\hline 7. & [EMIM][MeSO3] & cyclohexane & $\begin{array}{l}\text { indoline, pyridine, } \\
\text { quinoline, } \\
\text { pyyrole }\end{array}$ & 7 \\
\hline 8. & $\begin{array}{l}{[\mathrm{EMIM}]\left[\mathrm{EtSO}_{4}\right]} \\
{[\mathrm{EMPY}]\left[\mathrm{EtSO}_{4}\right]} \\
{[\mathrm{EMIM}]\left[\mathrm{MeSO}_{3}\right]}\end{array}$ & $n$-hexadecane & $\begin{array}{l}\text { Pyridine, indoline, } \\
\text { quinoline, pyyrole }\end{array}$ & 8 \\
\hline 9. & $\begin{array}{l}{[\mathrm{BMIM}]\left[\mathrm{BF}_{4}\right]} \\
{[\mathrm{BMIM}]\left[\mathrm{PF}_{6}\right]} \\
{[\mathrm{BMIM}][\mathrm{Cl}]} \\
{[\mathrm{BMIM}][\mathrm{Br}]} \\
{[\mathrm{BDMIM}]\left[\mathrm{BF}_{4}\right]} \\
{[\mathrm{EPy}][\mathrm{BF} 4]}\end{array}$ & $n$-hexane & pyridine & 9 \\
\hline 10. & $\begin{array}{l}{[\mathrm{TBHPP}][\mathrm{Cl}]} \\
{[\mathrm{TBCEP}][\mathrm{Br}]}\end{array}$ & n-heptane & BT, DBT, DMDBT & 10 \\
\hline 11. & $\begin{array}{l}\mathrm{CoCl}_{2}-\mathrm{ChCl} / 2 \mathrm{PEG} \\
\mathrm{CoCl}_{2}-\mathrm{ChCl} / 2 \mathrm{EG} \\
\mathrm{CoCl}_{2}-\mathrm{ChCl} / 2 \text { Glycerol }\end{array}$ & n-octane & BT, DBT, 4,6-DMBT & 11 \\
\hline
\end{tabular}


$\mathrm{CoCl}_{2}-\mathrm{ChCl} / 2 \mathrm{Pr}$

\begin{tabular}{|c|c|c|c|c|}
\hline & $\begin{array}{l}\text { TBABr:EG }(1: 2) \\
\text { TBPBr:EG }(1: 2)\end{array}$ & $n$-hexadecane & $\begin{array}{l}\text { Pyridine, indoline, } \\
\text { quinoline, pyyrole }\end{array}$ & 12 \\
\hline 13. & TPABr:AceA (1:4) & $\begin{array}{l}\text { Toluene, } \\
\text { decane }\end{array}$ & $\begin{array}{l}\text { Thiophene, pyridine, } \\
\text { pyyrole }\end{array}$ & 13 \\
\hline 14 & $\begin{array}{l}\text { THAmBr:EG (2:1) } \\
\text { THAmBr:G }(2: 1)\end{array}$ & n-heptane & $\begin{array}{l}\text { Benzothiazole, } \\
\text { thiophene }\end{array}$ & 14 \\
\hline 15 . & $\begin{array}{l}\text { ChCl:MA }(1: 1) \\
\text { ChCl: GA }(1: 1) \\
\text { ChCl: PAA }(1: 2) \\
\text { ChCl: PPA }(1: 2) \\
\text { ChCl: Glycerol }(1: 2) \\
\text { ChCl: Urea }(1: 2) \\
\text { Glycerol: CA }(2: 1) \\
\text { Fructose:Malic Acid }(1: 1)\end{array}$ & n-heptane & Pyridine, carbazole & 15 \\
\hline 16. & MTPPBr: EG (1:4) & $\begin{array}{l}n \text {-heptane, } \\
n \text {-hexane }\end{array}$ & $\begin{array}{l}\text { Pyridine, } \\
\text { benzothiazole }\end{array}$ & 16 \\
\hline 17. & $\begin{array}{l}\text { TEACl:EG (1:2) } \\
\text { TEACl: Glycerol (1:2) } \\
\text { MTPPBr:EG (1:3) } \\
\text { MTPPBr: Glycerol (1:3) }\end{array}$ & n-hexane & thiophene & 17 \\
\hline & MTPPBr: EG (1:4) & $\begin{array}{l}\text { Toluene, } \\
n \text {-heptane }\end{array}$ & Quinoline, indoline & 18 \\
\hline 19. & TBABr: PEG (1:2) & $\begin{array}{l}\text { Cyclohexane, iso- } \\
\text { octane, } n \text {-decane, } \\
\text { toluene }\end{array}$ & $\begin{array}{l}\text { Thiophene, DBT, } \\
\text { DBT, 4-MDBT, 4,6- } \\
\text { DMDBT }\end{array}$ & 19 \\
\hline
\end{tabular}


Table S2. List of Screened DESs for COSMO-RS Study

\begin{tabular}{|c|c|c|c|}
\hline No. & DES & Abbreviation & Molar Ratio \\
\hline 1. & Tetramethylammonium chloride- glycerol & TMACl : G & $1: 2$ \\
\hline 2. & Tetramethylammonium chloride- ethylene glycol & TMACl : EG & $1: 2$ \\
\hline 3. & Tetramethylammonium chloride- phenylacetic acid & TMACl : PAA & $1: 2$ \\
\hline 4. & Tetrabutylammonium chloride- malonic acid & TBACl : MA & $1: 2$ \\
\hline 5. & Tetrabutylammonium chloride- glycerol & TBACl : G & $1: 2$ \\
\hline 6. & Tetrabutylammonium chloride- tetraethylene glycol & TBACl : TEG & $1: 2$ \\
\hline 7. & Tetrabutylammonium chloride- ethylene glycol & TBACl : EG & $1: 2$ \\
\hline 8. & Tetrabutylammonium chloride- phenylacetic acid & TBACl : PAA & $1: 2$ \\
\hline 9. & Tetrabutylammonium chloride- caproic acid & TBACl : CapA & $1: 2$ \\
\hline 10. & Tetrabutylammonium chloride- acetic acid & TBACl : AceA & $1: 2$ \\
\hline 11. & Tetrabutylphosphonium bromide- ethylene glycol & TBPBr : EG & $1: 2$ \\
\hline 12. & Tetrabutylammonium bromide- ethylene glycol & TBABr : EG & $1: 2$ \\
\hline 13. & Methyltriphenylphosphonium bromide- glycerol & MTPPBr : G & $1: 2$ \\
\hline 14. & Methyltriphenylphosphonium bromide- glycerol & MTPPBr: G & $1: 3$ \\
\hline 15. & Methyltriphenylphosphonium bromide- glycerol & MTPPBr : G & $1: 4$ \\
\hline 16. & Methyltriphenylphosphonium bromide- ethylene glycol & MTPPBr : EG & $1: 2$ \\
\hline 17. & Methyltriphenylphosphonium bromide- ethylene glycol & MTPPBr : EG & $1: 3$ \\
\hline 18. & Methyltriphenylphosphonium bromide- ethylene glycol & MTPPBr : EG & $1: 4$ \\
\hline 19. & Methyltriphenylphosphonium bromide- ethylene glycol & MTPPBr : EG & $1: 5$ \\
\hline 20. & Methyltriphenylphosphonium bromide- tetraethylene glycol & MTPPBr : TEG & $1: 3$ \\
\hline 21. & Methyltriphenylphosphonium bromide- tetraethylene glycol & MTPPBr : TEG & $1: 4$ \\
\hline 22. & Methyltriphenylphosphonium bromide- tetraethylene glycol & MTPPBr : TEG & $1: 5$ \\
\hline 23. & Ethyltriphenylphosphonium bromide- ethylene glycol & ETPPBr : EG & $1: 2$ \\
\hline 24. & choline chloride- urea & $\mathrm{ChCl}: \mathrm{U}$ & $1: 2$ \\
\hline 25. & choline chloride- ethylene glycol & $\mathrm{ChCl}: \mathrm{EG}$ & $1: 2$ \\
\hline 26. & choline chloride- glycerol & $\mathrm{ChCl}: \mathrm{G}$ & $1: 2$ \\
\hline 27. & choline chloride- 2,2,2-trifluoroacetamice & $\mathrm{ChCl}: \mathrm{TFA}$ & $1: 2$ \\
\hline 28. & choline chloride- phenyl propionic acid & $\mathrm{ChCl}: \mathrm{PPA}$ & $1: 1$ \\
\hline 29. & Choline chloride- levulinic acid & $\mathrm{ChCl}: \mathrm{LA}$ & $1: 2$ \\
\hline 30. & Choline chloride- xylitol & $\mathrm{ChCl}: \mathrm{X}$ & $1: 1$ \\
\hline 31. & Choline chloride- D-sorbitol & $\mathrm{ChCl}: \mathrm{DS}$ & $1: 1$ \\
\hline 32. & Choline chloride- D-Isosorbide & $\mathrm{ChCl}$ : DIS & $1: 2$ \\
\hline 33. & Choline chloride- malonic acid & $\mathrm{ChCl}: \mathrm{MA}$ & $1: 1$ \\
\hline 34. & Choline chloride- malonic acid & $\mathrm{ChCl}: \mathrm{MA}$ & $1: 2$ \\
\hline 35. & Ethylcholine chloride- urea & $\mathrm{EChCl}: \mathrm{U}$ & $1: 2$ \\
\hline 36. & Acethylcholine chloride- urea & $\mathrm{AChCl}: \mathrm{U}$ & $1: 2$ \\
\hline 37. & Chlorocholine chloride- urea & $\mathrm{ClChCl}: \mathrm{U}$ & $1: 2$ \\
\hline 38. & N-benzyl-2-hydroxy-N,N-dimethylethanaminium chloride- Urea & bhdMEAmCl : U & $1: 2$ \\
\hline 39. & $\mathrm{~N}, \mathrm{~N}$-diethyl ethanol ammonium chloride- glycerol & dEEAmCl : G & $1: 2$ \\
\hline 40. & $\mathrm{~N}, \mathrm{~N}$-diethyl ethanol ammonium chloride- glycerol & dEEAmCl : G & $1: 3$ \\
\hline 41. & $\mathrm{~N}, \mathrm{~N}$-diethyl ethanol ammonium chloride- glycerol & dEEAmCl : G & $1: 4$ \\
\hline 42. & $\mathrm{~N}, \mathrm{~N}$-diethyl ethanol ammonium chloride- ethylene gycol & dEEAmCl : EG & $1: 2$ \\
\hline 43. & $\mathrm{~N}, \mathrm{~N}$-diethyl ethanol ammonium chloride- ethylene gycol & dEEAmCl : EG & $1: 3$ \\
\hline 44. & $\mathrm{~N}, \mathrm{~N}$-diethyl ethanol ammonium chloride- ethylene gycol & dEEAmCl : EG & $1: 4$ \\
\hline
\end{tabular}


Table S3. Chemical structure of cations used in this work.

\begin{tabular}{ccc} 
Name & Chemical Formula \\
\hline Choline & $\mathrm{C}_{5} \mathrm{H}_{15} \mathrm{NO}$ \\
\hline TMA & $\mathrm{C}_{4} \mathrm{H}_{12} \mathrm{~N}$ & SBP \\
\hline TMTPP & $\mathrm{C}_{16} \mathrm{H}_{36} \mathrm{~N}$
\end{tabular}


Table S4. Standard deviation (STDEV) on measured solubilities of hexadecane (1) / multisolute (2) mixture with TBPBr:EG (1:2) (3) for mole fractions x: Top layer

\begin{tabular}{|c|c|c|c|c|c|c|c|c|}
\hline \multicolumn{3}{|c|}{ Mole fraction } & \multicolumn{3}{|c|}{ Average mole fraction } & \multicolumn{3}{|c|}{ STDEV mole fraction } \\
\hline $\mathbf{x}_{1}$ & $\mathbf{x}_{2}$ & $\mathbf{x}_{3}$ & $\mathbf{x}_{1}$ & $\mathbf{x}_{2}$ & $\mathbf{x}_{3}$ & $\mathbf{x}_{1}$ & $\mathbf{x}_{2}$ & $\mathbf{x}_{3}$ \\
\hline 0.9875 & 0.0125 & 0.0000 & & & & & & \\
\hline 0.9877 & 0.0123 & 0.0000 & 0.9876 & 0.0124 & 0.0000 & 0.0001 & 0.0001 & 0.0000 \\
\hline 0.9877 & 0.0123 & 0.0000 & & & & & & \\
\hline 0.9762 & 0.0238 & 0.0000 & & & & & & \\
\hline 0.9758 & 0.0242 & 0.0000 & 0.9760 & 0.0240 & 0.0000 & 0.0002 & 0.0002 & 0.0000 \\
\hline 0.9760 & 0.0240 & 0.0000 & & & & & & \\
\hline 0.9592 & 0.0408 & 0.0000 & & & & & & \\
\hline 0.9605 & 0.0395 & 0.0000 & 0.9597 & 0.0403 & 0.0000 & 0.0007 & 0.0007 & 0.0000 \\
\hline 0.9595 & 0.0405 & 0.0000 & & & & & & \\
\hline 0.9498 & 0.0502 & 0.0000 & & & & & & \\
\hline 0.9499 & 0.0501 & 0.0000 & 0.9497 & 0.0503 & 0.0000 & 0.0003 & 0.0003 & 0.0000 \\
\hline 0.9494 & 0.0506 & 0.0000 & & & & & & \\
\hline 0.9108 & 0.0892 & 0.0000 & & & & & & \\
\hline 0.9139 & 0.0861 & 0.0000 & 0.9122 & 0.0878 & 0.0000 & 0.0015 & 0.0015 & 0.0000 \\
\hline 0.9120 & 0.0880 & 0.0000 & & & & & & \\
\hline 0.8896 & 0.1104 & 0.0000 & & & & & & \\
\hline 0.8917 & 0.1083 & 0.0000 & 0.8911 & 0.1089 & 0.0000 & 0.0013 & 0.0013 & 0.0000 \\
\hline 0.8918 & 0.1082 & 0.0000 & & & & & & \\
\hline
\end{tabular}


Table S5. Standard deviation (STDEV) on measured solubilities of hexadecane (1) / multisolute (2) mixture with TBPBr:EG (1:2) (3) for mole fractions x: Bottom layer

\begin{tabular}{cccccccccc}
\hline \multicolumn{7}{c}{ Mole fraction } & \multicolumn{7}{c}{ Average mole fraction } & \multicolumn{3}{c}{ STDEV mole fraction } \\
\hline $\mathbf{x}_{\mathbf{1}}$ & $\mathbf{x}_{\mathbf{2}}$ & $\mathbf{x}_{\mathbf{3}}$ & $\mathbf{x}_{\mathbf{1}}$ & $\mathbf{x}_{\mathbf{2}}$ & $\mathbf{x}_{\mathbf{3}}$ & $\mathbf{x}_{\mathbf{1}}$ & $\mathbf{x}_{\mathbf{2}}$ & $\mathbf{x}_{\mathbf{3}}$ \\
\hline 0.0123 & 0.1012 & 0.8865 & & & & & & \\
0.0122 & 0.1002 & 0.8876 & 0.0122 & 0.1014 & 0.8864 & 0.0000 & 0.0013 & 0.0013 \\
0.0122 & 0.1028 & 0.8850 & & & & & & \\
0.0055 & 0.1477 & 0.8468 & & & & & & \\
0.0055 & 0.1477 & 0.8468 & 0.0055 & 0.1485 & 0.8460 & 0.0000 & 0.0014 & 0.0014 \\
0.0055 & 0.1501 & 0.8444 & & & & & & \\
0.0054 & 0.2017 & 0.7929 & & & & & & \\
0.0056 & 0.2032 & 0.7913 & 0.0055 & 0.2030 & 0.7916 & 0.0001 & 0.0012 & \\
0.0055 & 0.2040 & 0.7905 & & & & & & \\
0.0061 & 0.2604 & 0.7334 & & & & & & \\
0.0063 & 0.2508 & 0.7429 & 0.0062 & 0.2593 & 0.7345 & 0.0002 & 0.0081 & \\
0.0060 & 0.2668 & 0.7272 & & & & & & \\
0.0074 & 0.3062 & 0.6864 & & & & & & \\
0.0073 & 0.3072 & 0.6854 & 0.0074 & 0.3071 & 0.6856 & 0.0001 & 0.0008 & \\
0.0074 & 0.3077 & 0.6849 & & & & & & & \\
0.0086 & 0.3975 & 0.5939 & & & & & & & \\
0.0086 & 0.3936 & 0.5978 & 0.0086 & 0.3956 & 0.5958 & 0.0000 & 0.0020 \\
0.0086 & 0.3958 & 0.5955 & & & & & & \\
\hline
\end{tabular}


Table S6. Standard deviation (STDEV) on measured solubilities of hexadecane (1) / multisolute (2) mixture with TBABr:EG (1:2) (3) for mole fractions x: Top layer

\begin{tabular}{|c|c|c|c|c|c|c|c|c|}
\hline \multicolumn{3}{|c|}{ Mole fraction } & \multicolumn{3}{|c|}{ Average mole fraction } & \multicolumn{3}{|c|}{ STDEV mole fraction } \\
\hline $\mathbf{x}_{1}$ & $\mathbf{x}_{2}$ & $\mathbf{x}_{3}$ & $\mathbf{x}_{1}$ & $\mathbf{x}_{2}$ & $\mathbf{x}_{3}$ & $\mathbf{x}_{1}$ & $\mathbf{x}_{2}$ & $\mathbf{x}_{3}$ \\
\hline 0.9868 & 0.0132 & 0.0000 & & & & & & \\
\hline 0.9876 & 0.0124 & 0.0000 & 0.9873 & 0.0127 & 0.0000 & 0.0005 & 0.0005 & 0.0000 \\
\hline 0.9876 & 0.0124 & 0.0000 & & & & & & \\
\hline 0.9778 & 0.0222 & 0.0000 & & & & & & \\
\hline 0.9789 & 0.0211 & 0.0000 & 0.9786 & 0.0214 & 0.0000 & 0.0007 & 0.0007 & 0.0000 \\
\hline 0.9792 & 0.0208 & 0.0000 & & & & & & \\
\hline 0.9676 & 0.0324 & 0.0000 & & & & & & \\
\hline 0.9691 & 0.0309 & 0.0000 & 0.9686 & 0.0314 & 0.0000 & 0.0009 & 0.0009 & 0.0000 \\
\hline 0.9693 & 0.0307 & 0.0000 & & & & & & \\
\hline 0.9451 & 0.0549 & 0.0000 & & & & & & \\
\hline 0.9478 & 0.0522 & 0.0000 & 0.9470 & 0.0530 & 0.0000 & 0.0016 & 0.0016 & 0.0000 \\
\hline 0.9480 & 0.0520 & 0.0000 & & & & & & \\
\hline 0.9390 & 0.0610 & 0.0000 & & & & & & \\
\hline 0.9421 & 0.0579 & 0.0000 & 0.9411 & 0.0589 & 0.0000 & 0.0018 & 0.0018 & 0.0000 \\
\hline 0.9422 & 0.0578 & 0.0000 & & & & & & \\
\hline 0.9109 & 0.0891 & 0.0000 & & & & & & \\
\hline 0.9111 & 0.0889 & 0.0000 & 0.9107 & 0.0893 & 0.0000 & 0.0006 & 0.0006 & 0.0000 \\
\hline 0.9101 & 0.0899 & 0.0000 & & & & & & \\
\hline
\end{tabular}


Table S7. Standard deviation (STDEV) on measured solubilities of hexadecane (1) / multisolute (2) mixture with TBABr:EG (1:2) (3) for mole fractions x: Bottom layer

\begin{tabular}{ccccccccc}
\hline \multicolumn{7}{c}{ Mole fraction } & \multicolumn{7}{c}{ Average mole fraction } & \multicolumn{3}{c}{ STDEV mole fraction } \\
\hline $\mathbf{x}_{\mathbf{1}}$ & $\mathbf{x}_{\mathbf{2}}$ & $\mathbf{x}_{\mathbf{3}}$ & $\mathbf{x}_{\mathbf{1}}$ & $\mathbf{x}_{\mathbf{2}}$ & $\mathbf{x}_{\mathbf{3}}$ & $\mathbf{x}_{\mathbf{1}}$ & $\mathbf{x}_{\mathbf{2}}$ & $\mathbf{x}_{\mathbf{3}}$ \\
\hline 0.0470 & 0.0815 & 0.8715 & & & & & & \\
0.0455 & 0.0699 & 0.8846 & 0.0459 & 0.0728 & 0.8813 & 0.0010 & 0.0076 & 0.0086 \\
0.0451 & 0.0671 & 0.8878 & & & & & & \\
0.0379 & 0.1157 & 0.8464 & & & & & & \\
0.0373 & 0.1155 & 0.8472 & 0.0374 & 0.1152 & 0.8474 & 0.0005 & 0.0008 & 0.0012 \\
0.0369 & 0.1143 & 0.8488 & & & & & & \\
0.0359 & 0.1439 & 0.8202 & & & & & & \\
0.0346 & 0.1437 & 0.8217 & 0.0351 & 0.1426 & 0.8222 & 0.0007 & 0.0020 & \\
0.0349 & 0.1403 & 0.8248 & & & & & & \\
0.0253 & 0.2013 & 0.7734 & & & & & & \\
0.0255 & 0.2070 & 0.7675 & 0.0254 & 0.2046 & 0.7699 & 0.0001 & 0.0031 \\
0.0255 & 0.2055 & 0.7690 & & & & & & \\
0.0224 & 0.2322 & 0.7454 & & & & & & \\
0.0225 & 0.2400 & 0.7375 & 0.0224 & 0.2375 & 0.7401 & 0.0001 & 0.0046 & 0.0046 \\
0.0223 & 0.2402 & 0.7374 & & & & & & \\
0.0280 & 0.2991 & 0.6728 & & & & & & \\
0.0234 & 0.2789 & 0.6978 & 0.0249 & 0.2845 & 0.6905 & 0.0027 & 0.0127 & \\
0.0234 & 0.2757 & 0.7009 & & & & & & \\
\hline
\end{tabular}


Table S8. Distribution Coefficient (D) and extraction efficiency $(\alpha)$ of individual solutes*

\begin{tabular}{cccccc}
\hline $\boldsymbol{D}_{\mathbf{2}}$ & $\boldsymbol{D}_{\mathbf{3}}$ & $\boldsymbol{D}_{\mathbf{4}}$ & $\boldsymbol{\alpha}_{\mathbf{2}},(\%)$ & $\boldsymbol{\alpha}_{\mathbf{3}},(\%)$ & $\boldsymbol{\alpha}_{\mathbf{4}},(\%)$ \\
\hline & & & $\mathrm{TBPBr}: \mathrm{EG}$ & \\
8.00 & 44.00 & 2.57 & 88.9 & 97.8 & 72.0 \\
7.00 & 60.00 & 1.73 & 87.5 & 98.4 & 63.4 \\
5.87 & 80.00 & 1.40 & 85.4 & 98.8 & 58.3 \\
6.21 & 96.00 & 1.48 & 86.1 & 99.0 & 59.7 \\
4.93 & 115.00 & 0.91 & 83.1 & 99.1 & 47.7 \\
4.19 & 73.00 & 1.08 & 80.7 & 98.6 & 51.9 \\
& & & TBABr:EG & & \\
7.00 & 30.00 & 1.88 & 87.5 & 96.8 & 65.2 \\
6.43 & 48.00 & 1.62 & 86.5 & 98.0 & 61.8 \\
5.70 & 59.00 & 1.40 & 85.1 & 98.3 & 58.3 \\
4.88 & 83.00 & 1.11 & 83.0 & 98.8 & 52.7 \\
5.76 & 95.00 & 1.10 & 85.2 & 99.0 & 52.3 \\
3.03 & 113.00 & 1.10 & 75.2 & 99.1 & 52.4 \\
\hline
\end{tabular}

$*_{2,3}$ and ${ }_{4}$ represent pyridine, indoline and DBT solutes, respectively.

Table S9. Selectivity (S) and extraction affinity ( $\beta$ ) of individual solutes*

\begin{tabular}{cccccc}
\hline $\boldsymbol{S}_{\mathbf{2}}$ & $\boldsymbol{S}_{\mathbf{3}}$ & $\boldsymbol{S}_{\mathbf{4}}$ & $\boldsymbol{\beta}_{\mathbf{2}},(\%)$ & $\boldsymbol{\beta}_{\mathbf{3}},(\%)$ & $\boldsymbol{\beta}_{\mathbf{4}}, \mathbf{( \% )}$ \\
\hline & & \multicolumn{3}{c}{ TBPBr:EG } & \\
658.7 & 3622.7 & 211.7 & 99.85 & 99.97 & 99.53 \\
1138.7 & 9760.0 & 282.0 & 99.91 & 99.99 & 99.65 \\
1126.4 & 15360.0 & 268.8 & 99.91 & 99.99 & 99.63 \\
983.3 & 15200.0 & 234.9 & 99.90 & 99.99 & 99.58 \\
642.1 & 14982.9 & 119.1 & 99.84 & 99.99 & 99.17 \\
414.4 & 7227.0 & 106.6 & 99.76 & 99.99 & 99.07 \\
& & & TBABr:EG & & \\
150.2 & 643.7 & 40.2 & 99.34 & 99.84 & 97.57 \\
170.1 & 1270.1 & 42.7 & 99.42 & 99.92 & 97.71 \\
157.8 & 1633.5 & 38.8 & 99.37 & 99.94 & 97.48 \\
184.9 & 3144.0 & 42.2 & 99.46 & 99.97 & 97.69 \\
246.6 & 4063.4 & 46.9 & 99.60 & 99.98 & 97.91 \\
110.3 & 4117.7 & 40.2 & 99.10 & 99.98 & 97.57 \\
\hline
\end{tabular}

$*_{2,3}$ and ${ }_{4}$ represent pyridine, indoline and DBT solutes, respectively. 


\section{List of figures}

Figure S1. GC Calibration curves of pyridine, indoline, DBT/dodecane systems

Figure S2. ${ }^{1} \mathrm{H}$ NMR spectra of TBABr:EG DES (1:2, molar ratio) in $\mathrm{CDCl}_{3}$ : (a) pure DES; (b) hydrocarbon rich phase (Top Layer).

Figure S3. ${ }^{1} \mathrm{H}$ NMR spectra of TBPBr:EG DES (1:2, molar ratio) in $\mathrm{CDCl}_{3}$ :(a) pure DES; (b) hydrocarbon rich phase (Top Layer)

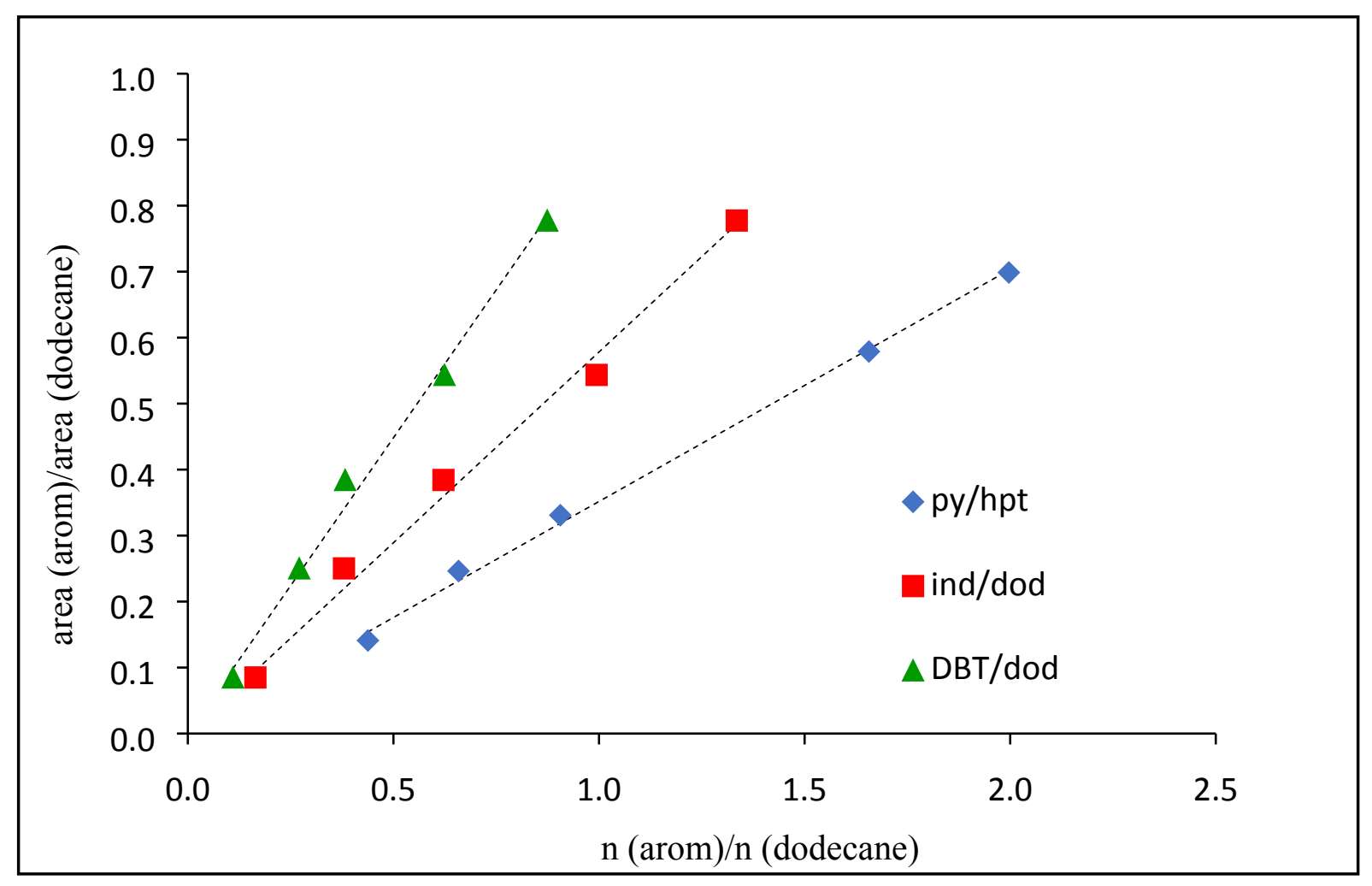

Figure S1. GC Calibration curves of pyridine, indoline, DBT/dodecane systems 


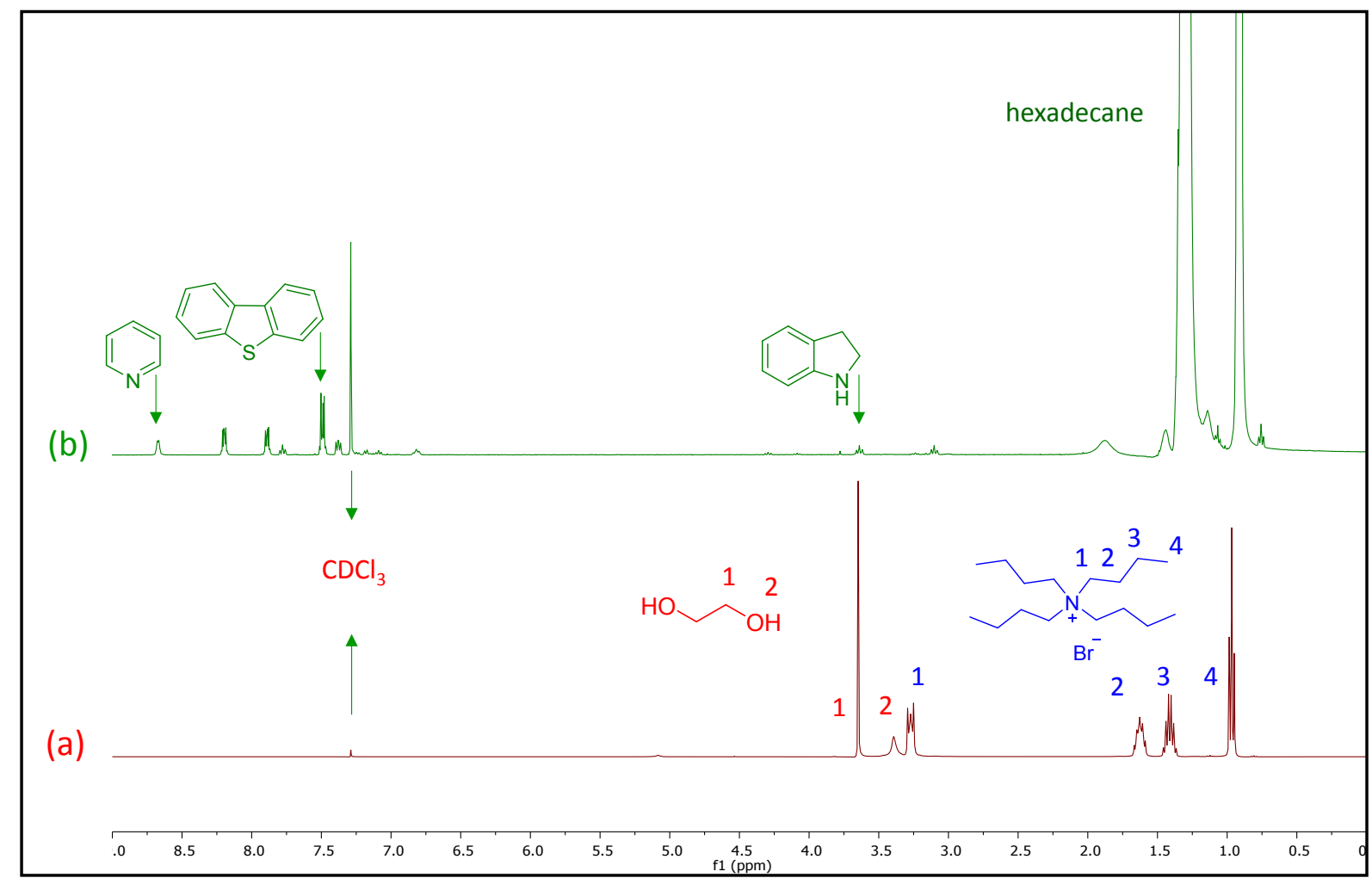

Figure S2. ${ }^{1} \mathrm{H}$ NMR spectra of TBABr:EG DES (1:2, molar ratio) in $\mathrm{CDCl}_{3}$ : (a) pure DES; (b) hydrocarbon rich phase (Top Layer). 


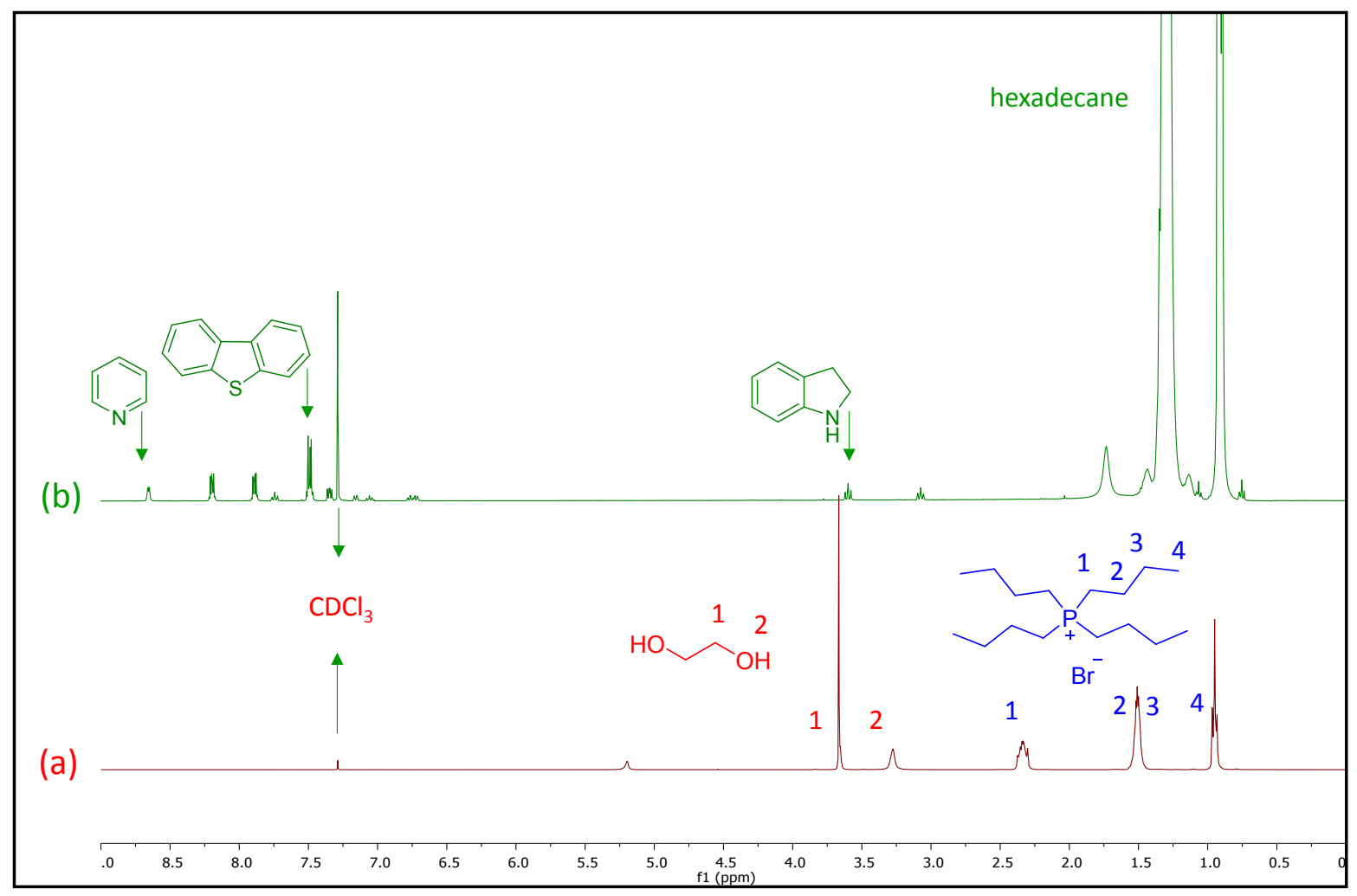

Figure S3. ${ }^{1} \mathrm{H}$ NMR spectra of TBPBr:EG DES (1:2, molar ratio) in $\mathrm{CDCl}_{3}$ :(a) pure DES; (b) hydrocarbon rich phase (Top Layer) 


\section{References}

1. El Sayed, H. A.; El Naggar, A. M. A.; Heakal, B. H.; Ahmed, N. E.; Said, S.; AbdelRahman, A. A. H., Deep catalytic desulphurization of heavy gas oil at mild operating conditions using self-functionalized nanoparticles as a novel catalyst. Fuel 2017, 209, 127-131.

2. $\quad$ Balinge, K. R.; Khiratkar, A. G.; Krishnamurthy, M.; Patle, D. S.; K, C. K.; Bhagat, P. R., Deep-desulfurization of the petroleum diesel using the heterogeneous carboxyl functionalized poly-ionic liquid. Resour. Effic. Technol. 2016, 2, S105-S113.

3. Tang, X.-d.; Zhang, Y.-f.; Li, J.-j.; Zhu, Y.-q.; Qing, D.-y.; Deng, Y.-X., Deep Extractive Desulfurization with Arenium Ion Deep Eutectic Solvents. Ind. Eng. Chem. Res 2015, 54 (16), 4625-4632.

4. Kang, L.; Liu, H.; He, H.; Yang, C., Oxidative desulfurization of dibenzothiophene using molybdenum catalyst supported on Ti-pillared montmorillonite and separation of sulfones by filtration. Fuel 2018, 234, 1229-1237.

5. Shah, S. S.; Ahmad, I.; Ahmad, W.; Ishaq, M.; Gul, K.; Khan, R.; Khan, H., Study on adsorptive capability of acid activated charcoal for desulphurization of model and commercial fuel oil samples. J. Environ. Chem. Eng. 2018, 6 (4), 4037-4043.

6. Gao, S.; Li, J.; Chen, X.; Abdeltawab, A. A.; Yakout, S. M.; Yu, G., A combination desulfurization method for diesel fuel: Oxidation by ionic liquid with extraction by solvent. Fuel 2018, 224, 545-551.

7. Salleh, M. Z. M.; Hadj-Kali, M. K.; Hizaddin, H. F.; Ali Hashim, M., Extraction of nitrogen compounds from model fuel using 1-ethyl-3-methylimidazolium methanesulfonate. Sep. Purif. Technol. 2018, 196, 61-70.

8. Hizaddin, H. F.; Hadj-Kali, M. K.; Ramalingam, A.; Hashim, M. A., Extraction of nitrogen compounds from diesel fuel using imidazolium- and pyridinium-based ionic liquids: Experiments, COSMO-RS prediction and NRTL correlation. Fluid Phase Equilib. 2015, 405, 55-67.

9. Yao, H.; Yang, D.; Li, C.; Wang, E., Intensification of water on the extraction of pyridine from n-hexane using ionic liquid. Chem. Eng. Process.: Process Intensif. 2018, 130, 61-66.

10. Moghadam, F. R.; Azizian, S.; Bayat, M.; Yarie, M.; Kianpour, E.; Zolfigol, M. A., Extractive desulfurization of liquid fuel by using a green, neutral and task specific phosphonium ionic liquid with glyceryl moiety: A joint experimental and computational study. Fuel 2017, 208, 214-222.

11. Xu, H.; Zhang, D.; Wu, F.; Wei, X.; Zhang, J., Deep desulfurization of fuels with cobalt chloride-choline chloride/polyethylene glycol metal deep eutectic solvents. Fuel 2018, 225, 104110 .

12. Hizaddin, H. F.; Hadj-Kali, M. K.; Ramalingam, A.; Ali Hashim, M., Extractive denitrogenation of diesel fuel using ammonium- and phosphonium-based deep eutectic solvents. J. Chem. Thermodyn. 2016, 95, 164-173.

13. Chandran, D.; Khalid, M.; Walvekar, R.; Mubarak, N. M.; Dharaskar, S.; Wong, W. Y.; Gupta, T. C. S. M., Deep eutectic solvents for extraction-desulphurization: A review. J. Mol. Liq. 2019, 275, 312-322. 
14. Farghi, F.; Kaddami, M., Liquid-Liquid Equilibrium of the Quaternary Systems n-Octane or Decane + Toluene + Dimethyl Sulfoxide + Methanol or Ethanol at 298.15 K: Experimental Data and Correlation. J. Solut. Chem. 2018, 47.

15. Ali, M. C.; Yang, Q.; Fine, A. A.; Jin, W.; Zhang, Z.; Xing, H.; Ren, Q., Efficient removal of both basic and non-basic nitrogen compounds from fuels by deep eutectic solvents. Green Chem. 2016, 18 (1), 157-164.

16. Warrag, S. E. E.; Alli, R. D.; Kroon, M. C., Liquid-Liquid Equilibrium Measurements for the Extraction of Pyridine and Benzothiazole from n-Alkanes Using Deep Eutectic Solvents. $J$. Chem. Eng. Data 2019, 64 (11), 4882-4890.

17. Gouveia, A. S. L.; Oliveira, F. S.; Kurnia, K. A.; Marrucho, I. M., Deep Eutectic Solvents as Azeotrope Breakers: Liquid-Liquid Extraction and COSMO-RS Prediction. ACS Sustain. Chem. Eng 2016, 4 (10), 5640-5650.

18. Ferro, V. R.; de Riva, J.; Sanchez, D.; Ruiz, E.; Palomar, J., Conceptual design of unit operations to separate aromatic hydrocarbons from naphtha using ionic liquids. COSMO-based process simulations with multi-component "real" mixture feed. Chem. Eng. Res. Des. 2015, 94, 632-647.

19. Ahmed Rahma, W. S.; Mjalli, F. S.; Al-Wahaibi, T.; Al-Hashmi, A. A., Polymeric-based deep eutectic solvents for effective extractive desulfurization of liquid fuel at ambient conditions. Chem. Eng. Res. Des. 2017, 120, 271-283. 